\title{
Article \\ Effect of a Training Program on Hepatic Fat Content and Cardiometabolic Risk in Postmenopausal Women: The Randomized Controlled Trial
}

\author{
Joaquín Reverter-Masia ${ }^{1, * \mathbb{D}}$, Alvaro Pano-Rodriguez ${ }^{1}$, Jose Vicente Beltran-Garrido ${ }^{2} \mathbb{D}$, Albert Lecube $^{3,4}$, \\ Enric Sánchez ${ }^{3}$ iD and Vicenç Hernández-González ${ }^{1}$
}

Citation: Reverter-Masia, J.;

Pano-Rodriguez, A.;

Beltran-Garrido, J.V.; Lecube, A.;

Sánchez, E.; Hernández-González, V.

Effect of a Training Program on

Hepatic Fat Content and

Cardiometabolic Risk in

Postmenopausal Women: The

Randomized Controlled Trial. Appl.

Sci. 2021, 11, 6409. https://doi.org/

10.3390/app11146409

Academic Editor: Herbert Wagner

Received: 22 June 2021

Accepted: 7 July 2021

Published: 12 July 2021

Publisher's Note: MDPI stays neutral with regard to jurisdictional claims in published maps and institutional affiliations.

Copyright: (C) 2021 by the authors. Licensee MDPI, Basel, Switzerland. This article is an open access article distributed under the terms and conditions of the Creative Commons Attribution (CC BY) license (https:/ / creativecommons.org/licenses/by/ $4.0 /)$.
1 Research Group Human Movement, Departament de Didàctiques Específiques, University of Lleida, Av. de 1'Estudi General, 25001 Lleida, Spain; alvarodepano@udl.cat (A.P.-R.); vicenc.hernandez@udl.cat (V.H.-G.)

2 EUSES Health and Sport Sciences School, Rovira i Virgili University, 43870 Tarragona, Spain; jose.vicente@euseste.es

3 Endocrinology and Nutrition Department, Hospital Universitari Arnau de Vilanova, Obesity, Diabetes and Metabolism Research Group (ODIM), Institut de Recerca Biomèdica de Lleida (IRBLleida), Universitat de Lleida, 25198 Lleida, Spain; alecube.lleida.ics@gencat.cat (A.L.); esanchez@irblleida.cat (E.S.)

4 Centro de Investigación Biomédica en Red de Diabetes y Enfermedades Metabólicas Asociadas (CIBERDEM), Instituto de Salud Carlos III (ISCIII), 28029 Madrid, Spain

* Correspondence: reverter@didesp.udl.cat; Tel.: +34-973-70-65-01

Featured Application: This study analyzes the suitability of the WB-EMS as a method to improve Hepatic Fat Content and Cardiometabolic Risk in Postmenopausal Women.

Abstract: This 10-week randomized controlled trial investigates the effects of two different training modalities on hepatic fat content and cardiometabolic risk in sedentary postmenopausal women. Besides, alterations in physical fitness, hepatic fat content, and cardiometabolic risk will be associated with changes in those blood parameters that are usually modifiable by exercise training. Postmenopausal women ( $n=32 ; \sim 61$ years) were randomly assigned to one of the following treatment groups; (1) based on international exercise recommendations (EX group; $n=16$ ), (2) exercise plus whole-body electromyostimulation (EX+EMS group; $n=16)$. Cardiometabolic risk score was calculated based on the international diabetes federation's clinical criteria. Hepatic fat content was estimated using the fatty liver index. After the intervention, the cardiometabolic risk and the fatty liver index decreased, showing a higher impact on EX + WB-EMS. Physical fitness was assessed through aerobic and strength tests belonging to "Eurofit Testing Battery". In all of them, significant differences were observed $(p<0.001)$, though EX+ EMS experienced better improvements $(p<0.05)$. In conclusion, a 10-week exercise training program, especially with WB-EMS, triggered improvements in physical fitness and reduced cardiometabolic risk and hepatic fat content in sedentary postmenopausal women.

Keywords: whole-body electromyostimulation; postmenopausal; hepatic fat content; cardiometabolic risk

Trial Registration: ISRCTN15558857 last edited: 02/12/2019 (retrospectively registered)

\section{Introduction}

The strong link between physical activity and health is a widely studied issue in the literature from diverse points of view. A huge amount of studies try to explain the relationship between these two paradigms [1-3]. The benefit that physical activity has on the different systems and organs of the human body, as well as the improvements which it promotes in functional capacity, has been established $[4,5]$. Consequently, there 
is compelling evidence showing the healthy impact of regular physical activity on older adults [1,6]. Unfortunately, despite a demonstrated inverse relationship between moderate to vigorous physical activity and cardiovascular or functional diseases in older adults existing ( $\geq 50$ years), few of them meet the physical activity recommendations made by the World Health Organization (i.e., $150 \mathrm{~min}$ of moderate-intensity aerobic activity, $75 \mathrm{~min}$ of vigorous-intensity aerobic activity, or an equivalent combination of them in $10 \mathrm{~min}$ bouts, and muscle-strengthening activities, involving major muscle groups. It should be done on 2 or more days a week.) [7]. In fact, previous researches concluded that sedentary behaviors, such as TV viewing, motorized transport, or leisure-time sitting, have been shown to contribute to adverse health outcomes in older people, such as deteriorated body composition [4], hypertension [5], impaired glucose metabolism [8], altered lipid metabolism (i.e., raised plasma triglycerides, total cholesterol and low-density lipoprotein cholesterol (LDL), and reduced high-density lipoprotein cholesterol (HDL)) [9], low cardiorespiratory fitness [10], and low physical fitness [11].

Moreover, many studies have been carried out analyzing the effectiveness of some novel biomarkers to predict hepatic steatosis and monitor responses to therapies $[12,13]$. An extensive number of clinical techniques have been used for non-alcoholic fatty liver disease (NAFLD) diagnosis, but most of them are time-consuming, often very expensive, and unavailable in many laboratories [14,15]. Interestingly, the exercise-based diagnosis has been used as an important management strategy in this cohort [16]. It has been shown that fatty liver index (FLI) scores of 60 and above ( $>60)$ indicate NAFLD [17]. In addition, some studies reported the effect of vigorous exercise training interventions in reducing fatty liver $[18,19]$.

It is well established that physical activity and exercise training improve cardiovascular function. In men, data show that vascular function is better in life-long physically active subjects [20]. In contrast to the relatively well-documented effect of exercise training in men, there is a paucity of studies in the literature related to cardiovascular function in women [21]. It has been shown that flow-mediated dilation is improved after a period of exercise training in aged men, but not in age-matched postmenopausal women [22]. It was found in another cross-sectional study that life-long trained postmenopausal women express a similar vascular dysfunction as their sedentary counterparts [23].

Menopause is an inevitable milestone among middle-aged women. With women's increasing life expectancy, even in developing countries, one-third to one-half of a woman's lifetime can be spent being postmenopausal [24]. Therefore, the health issues of postmenopausal women have become a growing concern in the health-care community because, as a consequence of their endocrine status, changes in body composition and cardiometabolic syndrome have been reported $[25,26]$. Risk factors for cardiovascular disease include smoking, physical inactivity, age, metabolic syndrome, and loss of estrogen [21,26]. Estrogen has been shown to have a protective effect on the cardiovascular system in women and the risk for cardiovascular events increases markedly after the menopausal transition, when substantial hormonal changes occur, including the loss of estrogen production. The implementation of exercise training can be used as a safe prophylactic strategy to oppose deteriorations in the cardiac system [27]. Nevertheless, results from studies with postmenopausal women concerning the effect of physical activity on their physical fitness and cardiovascular health are inconsistent. The results have shown a lack of effect of a physical activity program on some occasions and beneficial effects on others [21,25].

High-intensity training has been positioned as an efficient alternative [28] to induce improvements on cardiometabolic health $[29,30]$ and muscular strength [31] simultaneously [32], offering potentially better results in older and less fit individuals [32]. Although high-intensity training has been considered the most popular time-efficient exercise methodology, new training tendencies are emerging. Several sports centers and hospitals have been recently using a new exercise technique called whole body electromyostimulation (WB-EMS). It consists of the application of a rectangular, biphasic and symmetrical current by using a suit in which electrodes are strategically placed. The direct electrical impulse 
produces muscle contraction by transcutaneous peripheral nerve stimulation [33]. Devices generally allow the simultaneous activation of thighs, arms, buttocks, abdomen, chest, lower back area, upper back area, wide back and with two auxiliary channels of free choice, with a total electrode area of $2800 \mathrm{~cm}^{2}$ [34]. The suitability of using WB-EMS as an intensity method to improve the physical condition of older women has been raised, since it guarantees sufficient effort in those people unable or unwilling to do so on their initiative [35].

A recent systematic review concluded that, at the present time, despite the existence of some studies focusing on the improvement of cardiovascular endurance [36] and strength [35,37-41], as well as cardiometabolic risk [38,42,43], there is little evidence regarding the effectiveness of training with WB-EMS [44]. Regarding this controversy, new studies whose protocols adequately conform to the scientific methodology should be carried out $[11,45]$. Besides, to the best of the authors' knowledge, no previous studies focused on the association between physical fitness, cardiometabolic risk and the detection of nonalcoholic fatty liver disease on postmenopausal women. Thus, the purpose of this study was to compare the influence of traditional training vs. training whole-body electromyostimulation on physical fitness, cardiometabolic risk and detection of non-alcoholic fatty liver disease in sedentary menopausal women, in a 10-week WB-EMS training program.

\section{Materials and Methods}

\subsection{Ethics Statement and Reporting Philosophy}

This study is part of a large project called INDEST2016 and was conducted from September to November 2018. Its protocol was approved by the Ethics Committee of the Arnau of Vilanova University Hospital, Lléida (Spain) (CEIC-1701), and complies with the latest revision of the Declaration of Helsinki. A full description of it is available at ISRCTNresistry (ISRCTN15558857); last edited: 02 December 2019, and is also accessible elsewhere [46]. Data related to physical fitness after 10 weeks of WB-EMS training (i.e., balance, strength, velocity, power, flexibility, agility, speed and resistance) have been published elsewhere [11,47]. In the present manuscript, we included the comparisons between pre to post 10 weeks focused on hepatic fat content and cardiometabolic risk. Written, informed consent was obtained from all potential participants before their inclusion in the project. The reporting has been done following the CONSORT guideline for standard items in interventional trials [48].

\subsection{Participants}

The experimental procedure of the study corresponded to a two-arm randomized trial with parallel groups. The randomization of the study sample was carried out by a random number computer generator [49]. The participants were randomized into two different groups. The first group conducted a voluntary exercise program with superimposed WB-EMS (EX + WB-EMS, $n=16$ and the 2nd group performed only voluntary exercise training $(\mathrm{EX}, n=16)$. The general study design consisted of fitness condition, body composition analysis and biochemical variables, before and after a 10-week training period (see Figure 1).

Thirty-four post-menopausal women living in Lleida (Spain) were recruited to voluntarily participate in this investigation. The recruitment period lasted from June to August 2018. Briefly, in a first step, they were contacted by a phone call to be informed about the nature of the project. All of them were invited to attend an informational meeting, where more details were given on the benefits and possible risks that their participation in the project might entail. Those who showed interest in their participation were recruited, according to the inclusion criteria. Inclusion criteria were as follows: (1) reported contraindications (i.e., total endoprosthesis, abdomen/groin hernia, epilepsy, and cardiac arrhythmia) for WB-EMS intervention, (2) sedentary status according to the scales provided by the Eurobarometer [50], (3) postmenopausal status (detailed below in a separated section). Participants were allocated and informed about their assigned arm by a phone call 
which was made by an external collaborator. They were also assured of their anonymity and the reporting of their views in aggregate form to protect their identities.

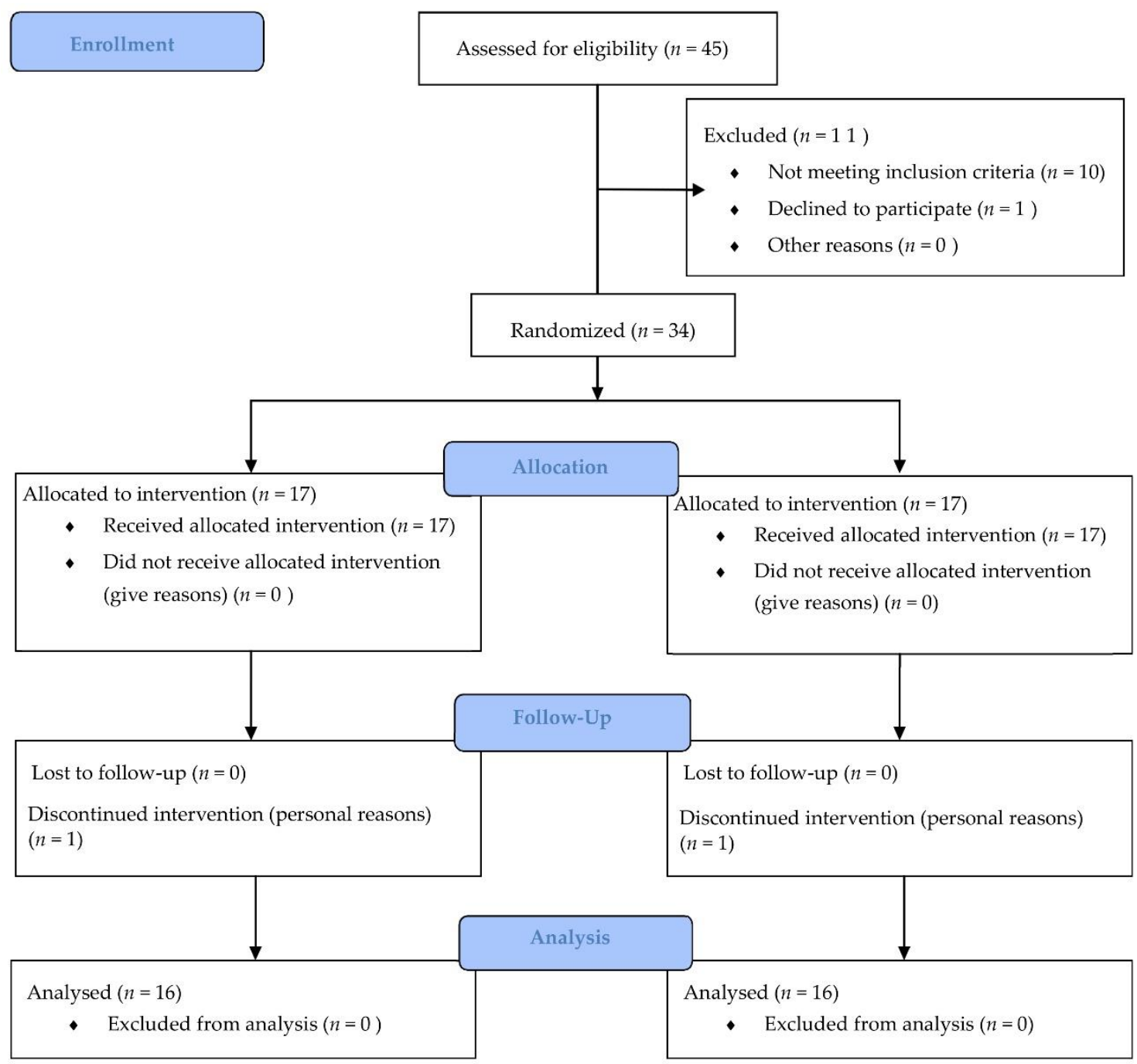

Figure 1. CONSORT Flow Diagram. Experimental protocol pre-pos test with control group; EX = Voluntary exercise group; $\mathrm{EX}+\mathrm{WB}-\mathrm{EMS}=$ Voluntary exercise with WB-EMS.

\subsection{Menopause Status}

Hormone assessments were performed from fasting serum samples taken between 8:00 and 10:00 AM. Serum was separated by centrifugation for $10 \mathrm{~min}$ at $2200 \times \mathrm{g}$. Systemic FSH levels were immunoassayed using IMMULITE 2000 XPi (Siemens Healthcare Diagnostics, Oxford, UK). Participants' menopause status was determined based on self-reported menstrual cycle.

Applying the categorization of Kovanen et al. 2018 [51], subjects were postmenopausal if no menstrual bleeding during the past 6 months, and the following cut values were applied FSH >30 IU/L. Participants self-reported their health problems, gynecologic status, and use of medications.

\subsection{Interventions}

Throughout the 10-week program, groups performed two training sessions each week with two rest days between sessions. The EX + WB-EMS group and the EX trained on differentiated days of the week so that one ignored the existence of the other. Both groups performed the same program consisting of endurance tasks and resistance strength 
exercises, but the EX + WB-EMS group also had a superimposed WB-EMS implemented during the training. The complete electrostimulation equipment, consisting of the suit and the electrostimulator device, does not weigh more than $1.5 \mathrm{~kg}$ and does not entail any limitation or discomfort for the movement of the body. Participants were asked not to make physical efforts outside the training program.

The sessions lasted $40 \mathrm{~min}$. Participants performed a $10 \mathrm{~min}$ warm-up by walking on a treadmill at a moderate speed. Subsequently, participants performed the resistance training protocol, which consisted of performing 3 multi-articular exercises involving push and pull actions (squat, deadlift, and bench press), as Aragão-Santos et al. 2018 [52] suggest as a recommended option in older people. The resistance training protocol lasted $10 \mathrm{~min}$, divided into 2 blocks of $5 \mathrm{~min}$. One block consisted of 10 sets of each exercise. Sets were composed of 2 repetitions with $2 \mathrm{~s}$ of eccentric and $1 \mathrm{~s}$ of concentric phase $(6 \mathrm{~s}$ in total per set). The intensity of the resistance training was $40 \%$ of the one-repetition maximum (1RM) obtained by an indirect measurement test [53]. Following the line of Wirtz et al. 2015 [54], the absolute load was increased by 5\% every two weeks to apply the principle of progressive overload. After strength exercises, participants performed a 10 min cardiovascular work on a treadmill, at a constant individualized speed, obtained from the talk test [55] (i.e., the highest speed they could walk while talking). The intensity of cardiovascular training was increased by $5 \%$ every week. Finally, the participants performed 10 min of stretching of the muscles of the whole body as a cooldown. At the end of each session, a scale was presented [56] with a range of 6 (no exertion at all) to 20 (maximal exertion), in which the participants of both groups recorded their internal training load perception. The assessment was always close to 15 (Hard).

The EX + WB-EMS group performed the resistance strength training with superimposed WB-EMS. A rectangular, bipolar compensated current of $6 \mathrm{~s}$ duration and $4 \mathrm{~s}$ rest was applied with a Wiemspro ${ }^{\circledR}$ electrostimulator (Malaga, Spain). The decision to use the Wiemspro ${ }^{\circledR}$ device in this study is based on its lightweight and short size, which makes it portable. Due to this, it is possible to wear the device attached to the body. This characteristic means that it does not impede or restrain the body's movements. Since there is evidence that a current frequency $\geq$ of $50 \mathrm{~Hz}$ is necessary to cause adaptations in strength training [57], during the strength exercises, a frequency of $55 \mathrm{~Hz}$ was applied with a $60 \%$ duty cycle (pulse width: leg and glute $350 \mu$ s, lumbar, rectus abdominis and latissimus dorsi $300 \mu \mathrm{s}$, trapezius $250 \mu \mathrm{s}$, chest $200 \mu \mathrm{s}$, and arms $150 \mu \mathrm{s}) 800 \mathrm{~ms}$ ascent ramp and descent ramp $500 \mathrm{~ms}[58,59]$. Taking into account the effectiveness of low frequencies of electrostimulation on the aerobic capacity [60], during cardiovascular training on the treadmill, the current applied was $7 \mathrm{~Hz}$, with a duty cycle of $100 \%$.

The fat modifies the transmission of the electrical stimuli into muscle [61]. Thus, the current intensity had to be normalized in the WB-EMS training. Following a procedure similar to that of Kemmler et al. 2010 [62], four levels of intensity perception of the electrical current (IPC) were established on a scale from 1 to 10 to control the perceived intensity produced by the application of the WB-EMS in the participants, being from 1 to 4 (mild), from 4 to 6 (moderate), from 6 to 8 (intense), and from 8 to 10 (pain). Participants gave constant information on the IPC during the session. In the first two weeks, training was conducted at a "moderate" IPC level to promote familiarization and adaptation to the WBEMS. In the remaining 8 weeks of the intervention, the intensity increased to an "intense" IPC level. When the same intensity level is maintained during a WB-EMS session, the IPC decreases over time. Hence, the intensity of the current increased gradually within the same session, without exceeding the level of IPC corresponding to that session.

Harms.: Adverse events including physical injuries were monitored by the instructors of the intervention and the responsible staff of the assessments. No harm occurred in the present study. 


\subsection{Assessments}

The assessments were carried out during the week before the training began (pre-test) and the week after the end of the intervention (post-test) in the sports center Ekke, located in Lleida (Spain). The data were recorded in a spreadsheet that was stored in an encrypted USB memory by an external collaborator, to guarantee the privacy of the participants. A blinded statistician had access to the final dataset of the study. He assessed the safety and validity of the research data. Participants were asked not to take any stimulants before assessment to avoid their influence on the results.

\subsubsection{Body Composition}

As secondary outcomes, the following parameters were measured: height, weight, body mass index (BMI) and waist perimeter. Height was determined with an accuracy of $0.10 \mathrm{~cm}$ with a SECA stadiometer (SECA, Hamburg, Germany). Participants were standing erect without shoes, with heels together and the head in the Frankfort horizontal plane. Body mass index was obtained using the formula: body weight/height2. To assess the perimeters, a measuring tape (CESCORF) was used [63].

All measurements were made in duplicate non-consecutively, and using the average value as the final value. All women were measured at the same time of the day for baseline and post-test, to avoid errors due to differences in hydration. All analyses were performed by a level I anthropometric technician certified by the International Society for the Advancement of Kineanthropometry (ISAK), as described in its Reference Manual [64].

\subsubsection{Blood Pressure}

Blood pressure was determined in the right arm after a $30 \mathrm{~min}$ rest in a supine position, using an Omrom ${ }^{\circledR}$ HEM 705 CP automatic monitor (OMROM Health-Care Co., Kyoto, Japan), following the recommendations of the European Heart Society [65]. A minimum of three measurements were taken $1 \mathrm{~min}$ apart, and the mean value was calculated.

\subsubsection{Blood Samples}

Venous blood samples were taken in fasting conditions (i.e., $~ 10 \mathrm{~h}$ ) from the antecubital vein and collected in ethylenediamine tetra-acetic acid-containing tubes using the Vacutainer SST system (Becton Dickinson, Plymouth, UK). All samples were centrifuged at $4000 \mathrm{rpm}$ for $7 \mathrm{~min}$ at $4{ }^{\circ} \mathrm{C}$, and aliquots of plasma were stored in $-80^{\circ} \mathrm{C}$ blood samples. Plasma glucose, total cholesterol, HDL-C, LDL, triglycerides, alanine transaminase (ALT), -glutamyl transferase (GGT), creatine kinase (CK) and creatinine were determined using an AU5800 absorption spectrophotometer (Beckman Coulter, Brea, CA, USA).

\subsubsection{Cardiometabolic Risk Score}

The International Diabetes Federation (IDF) has proposed as clinical criteria waist circumference, blood pressure, plasma glucose, HDL-C, and triglyceride concentrations, to define cardiometabolic risk [66]. Sex-specific cardiometabolic risk scores were calculated based on these criteria [67]. Each variable was standardized as follows: standardized value $=($ value - mean $) /$ standard deviation. The HDL-C standardized values were multiplied by -1 to represent increasing values as directly proportional to the risk score. The final score was determined as the sum of the five standardized scores divided by 5 . The cardiometabolic risk score is a continuous variable with a mean of 0 and a standard deviation of 1 by definition, with lower scores denoting a more favorable profile.

\subsubsection{Fatty Liver Index}

FLI is a validated surrogate marker of NAFLD [68]. This was calculated from the BMI, waist circumference, triglycerides, and GGT using the following equation [69]:

$\mathrm{FLI}=(\mathrm{e} 0.953 \times$ loge $($ triglycerides $)+0.139 \times \mathrm{BMI}+0.718 \times$ loge $(\mathrm{GGT})+0.053 \times$ waist circumference -15745$) /\left(1 \times 10^{0.953} \times\right.$ loge (triglycerides $)+0.139 \times \mathrm{BMI}+0.718$ 
$\times$ loge $($ GGT $)+0.053 \times$ waist circumference -15.745$) \times 100$. If FLI $<30$ no NAFLD; FLI > 60 NAFLD; FLI between 30-60 undetermined.

\subsubsection{Physical Fitness Measures}

The evaluation of the physical fitness was carried out using the tests modified and previously adapted from the "Senior Fitness Test Battery" and "Eurofit Testing Battery" [70].

\subsection{Statistical Procedures}

Data are presented in mean \pm standard deviation (SD). The assumption of normality was assessed by exploring the Q-Q plots and histogram of residuals. The homogeneity assumption was checked using Levene's test. The effectiveness of interventions was assessed by a 2-way mixed ANOVA. Group intervention ("EX+WB-EMS", "EX") was included as between-subject factor, time ("Pre", "Post") was included as the repeated with-in subject factor, and group $\times$ time was included to account for the interaction effects. Whenever a significant main effect or interaction was observed, Bonferroni's post hoc correction was used to aid interpretation. The statistician was blind to both groups during data analyses. The significance level was set at $\alpha=0.05$ for all tests. All statistical analyses were performed in JASP (version 0.11.1; JASP Team (2019), University of Amsterdam, the Netherlands).

\section{Results}

\subsection{Sample Characteristics}

The baseline characteristics of the study sample are shown in Table 1 . Non-statistically significant mean differences were shown between groups $(p>0.005)$.

Table 1. Summary of baseline characteristics of the sample.

\begin{tabular}{ccccc}
\hline Outcome & All & EX+WB-EMS & EX & $p$-Value \\
\hline Age (years) & $61.59 \pm 3.95$ & $62.94 \pm 3.32$ & $60.25 \pm 4.17$ & 0.053 \\
Body mass $(\mathrm{kg})$ & $67.44 \pm 10.84$ & $67.11 \pm 11.84$ & $67.78 \pm 10.12$ & 0.866 \\
Height $(\mathrm{cm})$ & $158.32 \pm 5.28$ & $156.69 \pm 5.02$ & $159.94 \pm 5.18$ & 0.081 \\
Body mass index & $26.91 \pm 4.11$ & $27.29 \pm 4.25$ & $26.54 \pm 4.08$ & 0.614 \\
$\left(\mathrm{~kg} / \mathrm{m}^{2}\right)$ & & & & \\
\hline
\end{tabular}

Data are presented as mean \pm SD. WB-EMS: Whole body electromyostimulation. EX + WB-EMS: Voluntary exercise with WB-EMS; EX: Voluntary exercise group.

\subsection{Body Composition}

The between-group changes in body composition outcomes are shown in Table 2. Non-significant main effects of time and group per time interactions were shown in all outcomes $(p>0.05)$.

Table 2. Summary of between-group changes in body composition outcomes.

\begin{tabular}{|c|c|c|c|c|c|c|}
\hline Outcome & Group & Week 0 & Week 10 & MD $[95 \% \mathrm{CI}]$ & $p$ (Time) & $p$ (Group $\times$ \\
\hline \multirow[t]{3}{*}{ Weight (kg) } & All & $67.44 \pm 10.84$ & $67.15 \pm 11.04$ & $0.29[-0.27,0.85]$ & 0.295 & 0.991 \\
\hline & EX + WB-EMS & $67.11 \pm 11.84$ & $66.82 \pm 12.10$ & $0.29[-0.80,1,38]$ & & \\
\hline & EX & $67.78 \pm 10.12$ & $67.49 \pm 10.27$ & $0.29[-0.80,1,38]$ & & \\
\hline \multirow[t]{3}{*}{$\operatorname{BMI}\left(\mathrm{kg} / \mathrm{m}^{2}\right)$} & All & $26.91 \pm 4.11$ & $26.81 \pm 4.16$ & $0.10[-0.13,0.34]$ & 0.367 & 0.853 \\
\hline & $\mathrm{EX}+\mathrm{WB}-\mathrm{EMS}$ & $27.29 \pm 4.25$ & $27.16 \pm 4.28$ & $0.13[-0.33,0.58]$ & & \\
\hline & EX & $26.54 \pm 4.08$ & $26.46 \pm 4.15$ & $0.08[-0.37,0.54]$ & & \\
\hline \multirow[t]{3}{*}{ Waist $(\mathrm{cm})$} & All & $82.02 \pm 16.99$ & $83.86 \pm 10.50$ & $-1.83[-6.85,2.84]$ & 0.428 & 0.283 \\
\hline & EX + WB-EMS & $84.13 \pm 10.88$ & $83.47 \pm 10.11$ & $0.66[-8.48,9.81]$ & & \\
\hline & EX & $79.90 \pm 21.64$ & $84.24 \pm 11.19$ & $-4.34[-13.48,4.80]$ & & \\
\hline
\end{tabular}

Data are presented as mean \pm SD. WB-EMS: Whole body electromyostimulation. EX+WB-EMS: exercise plus whole-body electrostimulation group; EX: exercise only group; EX + WB-EMS: Voluntary exercise with WB-EMS group; EX: Voluntary exercise group. Abbreviations: EX: exercise only group; MD: estimated mean difference; CI: confidence in-terval; $p: p$ Value. 


\subsection{Clinical Outcomes}

The between-group changes on clinical outcomes are shown in Table 3. A significant main effect of time was shown in systolic blood pressure $(p=0.028, \eta 2 p=0.19$, pre-post $\mathrm{MD}=6.88 \mathrm{mmHg} 95 \% \mathrm{CI}[0.80,12.96])$, diastolic blood pressure $(p=0.008, \eta 2 \mathrm{p}=0.26$, pre-post $\mathrm{MD}=3.60 \mathrm{mmHg} 95 \% \mathrm{CI}[1.03,6.17])$, mean blood pressure $(p=0.002, \eta 2 \mathrm{p}=0.34$, pre-post $\mathrm{MD}=4.69 \mathrm{mmHg} 95 \% \mathrm{CI}[1.95,7.43])$ and creatine kinase $(p=0.008, \eta 2 \mathrm{p}=0.21$, pre-post MD $=31.09 \mathrm{IU} / \mathrm{L} 95 \% \mathrm{CI}[8.74,53.45])$. Despite non-statistically significant interactions being shown in all clinical outcomes $(p>0.05)$, the EX + WB-EMS groups obtained higher pre-post estimated mean differences in systolic blood pressure, ALT, GGT and fatty liver index.

Table 3. Summary of between-group changes in clinical outcomes.

\begin{tabular}{|c|c|c|c|c|c|c|}
\hline Outcome & Group & Week 0 & Week 10 & $\begin{array}{c}\text { Estimated Mean } \\
\text { Difference }[95 \% \mathrm{CI}]\end{array}$ & $p$ (Time) & $p$ (Group $\times$ \\
\hline \multicolumn{7}{|l|}{ Blood pressure } \\
\hline \multirow{4}{*}{$\begin{array}{l}\text { Systolic blood } \\
\text { pressure } \\
(\mathrm{mm} \mathrm{Hg})\end{array}$} & & & & & & \\
\hline & All & $121.14 \pm 16.80$ & $127.00 \pm 20.76$ & $6.88[0.80,12.96]$ & 0.028 & 0.516 \\
\hline & EX + WB-EMS & $122.64 \pm 16.90$ & $131.46 \pm 21.88$ & $8.82[-4.05,21.69]$ & & \\
\hline & EX & $119.60 \pm 18.24$ & $124.53 \pm 18.00$ & $4.93[-6.09,15.95]$ & & \\
\hline \multirow{4}{*}{$\begin{array}{c}\text { Diastolic blood } \\
\text { pressure } \\
(\mathrm{mm} \mathrm{Hg})\end{array}$} & & & & & & \\
\hline & All & $73.32 \pm 7.89$ & $76.70 \pm 7.29$ & $3.60[1.03,6.17]$ & 0.008 & 0.211 \\
\hline & EX + WB-EMS & $74.00 \pm 7.94$ & $76.00 \pm 6.40$ & $2.00[-3.43,7.43]$ & & \\
\hline & EX & $72.33 \pm 8.40$ & $77.53 \pm 7.47$ & $5.20[0.55,9,85]$ & & \\
\hline \multirow{4}{*}{$\begin{array}{c}\text { Mean blood } \\
\text { pressure } \\
(\mathrm{mm} \mathrm{Hg})\end{array}$} & & & & & & \\
\hline & All & $89.26 \pm 9.61$ & $93.47 \pm 10.49$ & $4.69[1.95,7.43]$ & 0.002 & 0.755 \\
\hline & EX + WB-EMS & $90.21 \pm 8.81$ & $94.49 \pm 9.94$ & $4.27[-1.53,10.07]$ & & \\
\hline & EX & $88.09 \pm 10.89$ & $93.20 \pm 9.70$ & $5.11[0.14,10.08]$ & & \\
\hline \multicolumn{7}{|l|}{ Liver function } \\
\hline \multirow[t]{3}{*}{ ALT (IU/L) } & All & $24.72 \pm 16.04$ & $21.28 \pm 13.39$ & $-3.44[-10.31,3.43]$ & 0.315 & 0.203 \\
\hline & EX + WB-EMS & $30.81 \pm 20.35$ & $23.00 \pm 16.96$ & $-7.81[-21.25,5.63]$ & & \\
\hline & EX & $18.63 \pm 6.17$ & $19.56 \pm 8.76$ & $0.94[-12.50,14.38]$ & & \\
\hline \multirow[t]{3}{*}{ GGT (IU/L) } & All & $26.91 \pm 21.74$ & $22.56 \pm 13.04$ & $-4.34[-12.12,3.43]$ & 0.263 & 0.655 \\
\hline & EX + WB-EMS & $31.46 \pm 28.80$ & $25.50 \pm 16.55$ & $-6.06[-21.26,9.14]$ & & \\
\hline & EX & $22.25 \pm 10.04$ & $19.63 \pm 7.68$ & $-2.63[17.83,12.58]$ & & \\
\hline \multirow[t]{3}{*}{$\begin{array}{l}\text { Fatty liver } \\
\text { index }\end{array}$} & All & $30.70 \pm 27.67$ & $28.16 \pm 25.78$ & $-2.55[-6.79,1.70]$ & 0.230 & 0.381 \\
\hline & EX + WB-EMS & $34.05 \pm 29.11$ & $29.65 \pm 26.54$ & $-4.39[-12.70,3.91]$ & & \\
\hline & EX & $27.36 \pm 26.67$ & $26.66 \pm 25.78$ & $-0.70[-9.01,7.61]$ & & \\
\hline \multicolumn{7}{|l|}{$\begin{array}{l}\text { Markers of } \\
\text { overtraining }\end{array}$} \\
\hline \multirow[t]{3}{*}{$\begin{array}{l}\text { Creatine kinase } \\
\text { (CPK; IU/L) }\end{array}$} & All & $101.66 \pm 39.33$ & $132.75 \pm 67.21$ & $31.09[8.74,53.45]$ & 0.008 & 0.182 \\
\hline & EX + WB-EMS & $94.19 \pm 41.45$ & $140.25 \pm 81.71$ & $46.06[2.33,89.79]$ & & \\
\hline & EX & $109.13 \pm 36.87$ & $125.50 \pm 48.72$ & $16.13[-27.61,59.86]$ & & \\
\hline \multirow[t]{3}{*}{$\begin{array}{l}\text { Creatinine } \\
(\mathrm{mg} / \mathrm{dL})\end{array}$} & All & $0.71 \pm 0.10$ & $0.70 \pm 0.11$ & $-0.00[-0.03,0.02]$ & 0.812 & 0.812 \\
\hline & EX + WB-EMS & $0.71 \pm 0.10$ & $0.71 \pm 0.12$ & & & \\
\hline & EX & $0.70 \pm 0.10$ & $0.70 \pm 0.11$ & & & \\
\hline
\end{tabular}

Data are presented as mean \pm SD. WB-EMS: Whole body electromyostimulation. EX + WB-EMS: Voluntary exercise with WB-EMS; EX: Voluntary exercise group. Significant mean differences and $p$-values $(p \leq 0.05)$ are shown in bold. Abbreviations: ALT-Alanine transaminase; GGT—glutamyl transferase, EX: exercise only group; MD: mean difference; CI: confidence interval; $p: p$ Value. 


\subsection{Hematological Outcomes}

The between-group changes in hematological measures are shown in Table 4. A significant main effect of time was shown in glucose only $(p=0.015, \eta 2 p=0.18$, prepost $\mathrm{MD}=4.19 \mathrm{mmol} / \mathrm{L} 95 \% \mathrm{CI}[0.88,7.50])$. Even though non-statistically significant interactions were shown in all clinical outcomes $(p>0.05)$, the EX + WB-EMS groups obtained higher pre-post estimated mean differences in HDL, triglycerides, glucose and cardiometabolic risk score.

Table 4. Summary of between-group changes on hematological measures.

\begin{tabular}{|c|c|c|c|c|c|c|}
\hline Outcome & Group & Week 0 & Week 10 & $\begin{array}{c}\text { Estimated Mean } \\
\text { Difference }[95 \% \mathrm{CI}]\end{array}$ & $p$ (Time) & $p$ (Group $\times$ \\
\hline \multirow[t]{3}{*}{$\begin{array}{c}\text { Total } \\
\text { cholesterol } \\
(\mathrm{mg} / \mathrm{dL})\end{array}$} & All & $222.88 \pm 25.95$ & $218.88 \pm 31.10$ & $-4.00[-14.04,6.04]$ & 0.422 & 0.920 \\
\hline & EX + WB-EMS & $219.19 \pm 29.90$ & $215.69 \pm 37.15$ & $-3.50[-23.14,16.14]$ & & \\
\hline & EX & $226.56 \pm 21.65$ & $222.06 \pm 24.43$ & $-4.50[-24.14,15.14]$ & & \\
\hline \multirow[t]{3}{*}{ HDL (mmol/L) } & All & $64.78 \pm 12.88$ & $65.88 \pm 14.08$ & $1.09[-2.16,4.34]$ & 0.497 & 0.547 \\
\hline & EX + WB-EMS & $66.19 \pm 15.38$ & $68.25 \pm 15.64$ & $2.06[-4.29,8.42]$ & & \\
\hline & EX & $63.38 \pm 10.10$ & $63.50 \pm 12.37$ & $0.13[-6.23,6.48]$ & & \\
\hline \multirow[t]{3}{*}{$\mathrm{LDL}(\mathrm{mmol} / \mathrm{L})$} & All & $142.45 \pm 32.64$ & $135.67 \pm 25.70$ & $-6.78[-18.05,4.49]$ & 0.229 & 0.624 \\
\hline & EX + WB-EMS & $134.20 \pm 22.76$ & $130.15 \pm 28.67$ & $-4.05[-26.10,18.00]$ & & \\
\hline & EX & $150.70 \pm 39.23$ & $141.19 \pm 21.88$ & $-9.51[-31.56,12.54]$ & & \\
\hline \multirow{3}{*}{$\begin{array}{l}\text { Triglycerids } \\
(\mathrm{mmol} / \mathrm{L})\end{array}$} & All & $93.84 \pm 35.21$ & $86.97 \pm 23.22$ & $-6.88[.18 .28,4.53]$ & 0.228 & 0.903 \\
\hline & EX + WB-EMS & $94.00 \pm 41.79$ & $86.44 \pm 22.22$ & $-7.56[-29.88,14.75]$ & & \\
\hline & EX & $93.69 \pm 28.56$ & $87.50 \pm 24.91$ & $-6.19[-28.50,16.13]$ & & \\
\hline \multirow{3}{*}{$\begin{array}{l}\text { Glucose } \\
(\mathrm{mmol} / \mathrm{L})\end{array}$} & All & $90.78 \pm 10.52$ & $94.97 \pm 10.16$ & $4.19[0.88,7.50]$ & 0.015 & 0.848 \\
\hline & EX + WB-EMS & $91.38 \pm 11.88$ & $95.88 \pm 8.39$ & $4.50[-1.97,10.97]$ & & \\
\hline & EX & $90.19 \pm 9.30$ & $94.06 \pm 11.89$ & $3.88[-2.59,10.34]$ & & \\
\hline \multirow{3}{*}{$\begin{array}{l}\text { Cardiometabolic } \\
\text { risk score }\end{array}$} & All & $-0.26 \pm 0.88$ & $-0.07 \pm 0.52$ & $0.19[-0.13,0.51]$ & 0.235 & 0.574 \\
\hline & EX + WB-EMS & $-0.42 \pm 1.09$ & $-0.14 \pm 0.44$ & $0.28[-0.35,0.91]$ & & \\
\hline & EX & $-0.10 \pm 0.62$ & $0.00 \pm 0.59$ & $0.10[-0.53,0.73]$ & & \\
\hline
\end{tabular}

Data are presented as mean \pm SD. WB-EMS: Whole body electromyostimulation. EX + WB-EMS: Voluntary exercise with WB-EMS; EX: Voluntary exercise group. Significant mean differences and $p$-values $(p \leq 0.05)$ are shown in bold. Abbreviations: HDL-high-density lipoprotein cholesterol; LDL-low-density lipoprotein, EX: exercise only group; MD: mean difference; CI: confidence interval; $p$ : $p$ Value.

\subsection{Physical Fitness Outcomes}

The between-group changes on fitness measures are shown in Table 5. The data revealed a significant main effect of time $(p<0.001, \eta 2 p=0.81)$ and interaction $(p=0.025$, $\eta 2 p=0.18)$ in strength of right arm. Post-hoc tests revealed significant mean difference between the pre- and post-tests of both groups (EX+WB-EMS: 6.50 repetitions $95 \%$ CI [4.41, 8.59], $p_{\text {Bonferroni }}<0.001$; EX: 4.13 repetitions 95\% CI [2.03, 6.22], $\left.p_{\text {Bonferroni }}<0.001\right)$. However, non-statistically significant between-group differences were shown in either pretest or post-test (pre-test: 1.44 repetitions $95 \%$ CI $[-1.06,3.93], p_{\text {Bonferroni }}=0.723$; post-test: -0.93 repetitions $\left.95 \% \mathrm{CI}[-3.43,1.56], p_{\text {Bonferroni }}=1.000\right)$.

A significant main effect of time $(p<0.001, \eta 2 p=0.84)$ and interaction $(p=0.031$, $\eta 2 p=0.17)$ was obtained in strength of left arm. Post-hoc tests revealed a significant mean difference between pre and post-tests of both groups (EX+WB-EMS: 6.56 repetitions 95\% CI $[4.81,8.31], p_{\text {Bonferroni }}<0.001$; EX: 4.37 repetitions 95\% CI [2.63, 6.12], $\left.p_{\text {Bonferroni }}<0.001\right)$. However, non-statistically significant between-group differences were shown in either pretest or post-test (pre-test: 1.43 repetitions $95 \% \mathrm{CI}[-1.16,4.03], p_{\text {Bonferroni }}=0.800$; post-test: -0.75 repetitions $\left.95 \% \mathrm{CI}[-3.34,1.84], p_{\text {Bonferroni }}=1.000\right)$.

Finally, a significant main effect of time $(p<0.001, \eta 2 p=0.81)$ and interaction $(p>0.001$, $\eta 2 p=0.68)$ was obtained in the 6 min walk test. Post hoc tests revealed significant 
mean differences between pre- and post-tests of EX+WB-EMS group (155.54 m 95\% CI $\left.[122.99,188.08], p_{\text {Bonferroni }}<0.001\right)$, but not in the EX group $(25.66 \mathrm{~m} \mathrm{95 \%} \mathrm{CI}[-6.88,58.21]$, emphp $\left._{\text {Bonferroni }}=0.201\right)$. Statistically significant between-group differences were shown in post-test $\left(-117.63 \mathrm{~m} \mathrm{95 \%} \mathrm{CI}[-174.54,60.71]\right.$, $\left.p_{\text {Bonferroni }}<0.001\right)$, but not in pre-test $(12.25 \mathrm{~m}$ 95\% CI $\left.[-44.66,69.16], p_{\text {Bonferroni }}=1.0000\right)$.

Table 5. Summary of between-group changes on physical fitness measures.

\begin{tabular}{|c|c|c|c|c|c|c|}
\hline Outcome & Group & Week 0 & Week 10 & $\begin{array}{c}\text { Estimated Mean } \\
\text { Difference }[95 \% \mathrm{CI}]\end{array}$ & $p$ (Time) & $p\left(\begin{array}{c}\text { (Group } \times \\
\text { Time })\end{array}\right.$ \\
\hline \multirow[t]{2}{*}{$\begin{array}{l}\text { Strength right } \\
\text { arm (reps) }\end{array}$} & All & $15.53 \pm 2.57$ & $20.84 \pm 2.65$ & $5.31[4.24,6.38]$ & $<0.001$ & 0.031 \\
\hline & $\begin{array}{c}\mathrm{EX}+\mathrm{WB}-\mathrm{EMS} \\
\mathrm{EX}\end{array}$ & $\begin{array}{l}14.81 \pm 2.26 \\
16.25 \pm 2.72\end{array}$ & $\begin{array}{c}21.31 \pm 3.05 \\
20.375 \pm 2.19\end{array}$ & $\begin{array}{l}6.50[4.41,8.59] \\
4.13[2.03,6.22]\end{array}$ & & \\
\hline \multirow{2}{*}{$\begin{array}{l}\text { Strength left } \\
\text { arm (reps) }\end{array}$} & All & $15.78 \pm 2.46$ & $21.25 \pm 2.89$ & $5.47[4.58,6.36]$ & $<0.001$ & 0.018 \\
\hline & $\begin{array}{c}\mathrm{EX}+\mathrm{WB}-\mathrm{EMS} \\
\mathrm{EX}\end{array}$ & $\begin{array}{l}15.06 \pm 1.73 \\
16.50 \pm 2.90\end{array}$ & $\begin{array}{c}21.63 \pm 3.074 \\
20.88 \pm 2.73\end{array}$ & $\begin{array}{l}6.56[4.82,8.31] \\
4.38[2.63,6.12]\end{array}$ & & \\
\hline \multirow{2}{*}{$\begin{array}{l}6 \text { min walk } \\
\text { test }(\mathrm{m})\end{array}$} & All & $567.90 \pm 57.32$ & $658.50 \pm 82.74$ & $90.60[73.96,107.24]$ & $<0.001$ & $<0.001$ \\
\hline & $\begin{array}{c}\mathrm{EX}+\mathrm{WB}-\mathrm{EMS} \\
\mathrm{EX}\end{array}$ & $\begin{array}{l}561.78 \pm 54.58 \\
574.03 \pm 61.09\end{array}$ & $\begin{array}{l}717.31 \pm 59.91 \# \\
599.688 \pm 56.41\end{array}$ & $\begin{array}{c}155.54[122.99,188.08] \\
25.66[-6.88,58.21]\end{array}$ & & \\
\hline
\end{tabular}

Data are presented as mean \pm SD. WB-EMS: Whole body electromyostimulation. EX + WB-EMS: Voluntary exercise with WB-EMS; EX: Voluntary exercise group. Significant mean differences and $p$-values $(p \leq 0.05)$ are shown in bold. \# $p_{\text {Bonferroni }} \leq 0.05$ different to EX group values. EX: exercise only group; MD: mean difference; CI: confidence interval, $p: p$ Value.

\section{Discussion}

The main finding of this work is that a 10-week supervised exercise intervention produces effects in cardiometabolic risk, NAFLD parameters and physical fitness in sedentary postmenopausal women. It should be noted that, although EX and EX + WB-EMS experienced reductions in their cardiometabolic risk, a slightly higher effect in EX + WB-EMS can be observed. Besides, despite the exigent exercitation in this study, the $\mathrm{CK}$ values indicated the inexistence of rhabdomyolysis.

Body composition and distribution of the fat tissue experience changes over the years [71]. Mainly, the menopause stage favors fat accumulation. Thus, menopausal women often gain around $10 \%$ of their pre-menopause weight [72,73]. Following the line of the present study, some other research groups have found a prevalence of $80 \%$ of overweight and obesity in Spanish postmenopausal women [74-76]. Therefore, the determination of body composition is an important factor that should not be disregarded. The study of this variable may help in the improvement of medical knowledge in postmenopausal sedentary women. Waist circumference is a predictor of visceral fat located in the abdominal region [77]. Its measurement is an easy and low-cost procedure with relevant importance in population-based studies [78]. Waist circumference shows a high correlation with the majority of metabolic risk factors $[78,79]$. Some previous studies assessed the effects of WB-EMS on body composition of untrained old adult men and women finding statistical differences, but showing a high risk of bias because of the different and not comparable treatments applied to the study groups $[38,43,62,80-84]$. It impairs their data reliability. In the present study, EX + WB-EMS group did not show significant differences in waist circumference, weight, or IMC compared to the EX group as it happens in other studies where the body composition does not show differences $[36,41,85,86]$.

Many authors have studied the effect of moderate and intense intensity physical activity on blood pressure, obtaining results similar to ours [87]. Arterial hypertension is a chronic or persistent increase in systolic blood pressure (SBP) greater than or equal to $140 \mathrm{mmHg}$ and/or a diastolic blood pressure (DBP) greater than $90 \mathrm{mmHg} 1$. In our work, it is appreciated how these values are not exceeded. Perhaps in future work, it will be 
necessary to propose more extended aerobic tasks in order to obtain a significant drop in blood pressure, as stated by different authors [88,89].

Recently, WB-EMS is an effective tool for improving muscle strength outcome measurements in deconditioned subjects [11]. However, an excessive intensity in the first phase of a WB-EMS training program was related to some severe side effects. A few case reports have characterized substantial muscle damage and rhabdomyolysis following just one single training session [90-92]. In fact, recent studies showed an extreme increase in muscle damage markers, such as CK after a WB-EMS application [93,94]. In the present study, the results showed values considered normal in the literature [95]. An increase of CK values begins between 2 and $12 \mathrm{~h}$ after the muscular damage and then a progressive decrease is observed during the next five days if the exercise stops [96]. Muscular damage is usually accompanied by an increase of GGT and ALT, which could emulate hepatic damage [97]. In the present study, the GGT and ALT decreased, which could confirm, together with the CK values, that no subject of this study suffered rhabdomyolysis. As indicated by the literature, the correct application of the training program by professionals is important for safe and effective training [91,93]. In this study, the training protocols were supervised by two instructors graduated in Physical Activity and Sports Sciences and post-graduated in Sports Training. Their academic formation and wide experience in WB-EMS training guaranteed the correct training load administration, which led to safety training with a decreasing GGT and ALT.

We observed changes in triglycerides in both groups, which is in agreement with the results of previous studies showing a positive influence of concurrent training in the blood triglyceride values $[98,99]$. This could be explained by the idea that combined strength and aerobic training increases the rate of fat oxidation, also presenting triglycerides as an important source of fuel for this type of exercise. Exercise would lead to triglycerides consumed by muscle tissue, and increases lipoprotein lipase (LPL), which would result in more triglycerides hydrolysis [100].

Recent studies have shown that low skeletal muscle mass can contribute to NAFLD, mostly in postmenopausal women [101,102]. The FLI was described by Bedogni et al., 2006 [68] for the diagnosis of patients with NAFLD, and includes BMI, abdominal circumference, triglycerides and GGT, with values ranging from 0 to 100 . NAFLD, which is associated with cardiovascular disease, is characterized by the deposition of free fatty acids and triglycerides in the cytoplasm of hepatocytes, in the form of large vacuoles, in patients without toxic alcohol consumption, and not associated with other liver diseases $[103,104]$. It is also a predictor of type 2 diabetes [105]. Thus, the role of FLI as an independent marker of cardiovascular disease, without cardiovascular risk factors, is interesting. According to the results of three studies, when FLI value is higher than 60, it indicates an increased cardiovascular risk [106,107]. In our work, we found that FLI improved significantly after 10 weeks of training in both groups. The whole sample ended up with FLI below 30, which is the value recommended by scientific societies as a predictor of good health [108]. It should be noted that, in the case of the EX + WB-EMS group, the effect size was much larger than in the EX group. The improvement of some subject's FLI in this study may reflect the reduction of ectopic fat deposits that are responsible for the improvement of insulin resistance, these results would confirm those found by other authors [106]. Some studies suggested positive effects of an exercise intervention on liver metabolic signaling pathways, such as improved oxidative enzyme activity, increased fatty acid oxidation and reduced intracellular lipid accumulation in the liver [109]. Barsalani et al. 2013 illustrated that significant changes can be observed in FLI response following lifestyle intervention such as exercise training and diets [110]. It has been established that the improvement of the FLI is linked to the intensity of the exercise [111]. The valuable information provided by our study is that the EX + WB-EMS group showed remarkably better improvement compared to the EX group, so we suspect that WB-EMS training could be an adequate exercise methodology for the treatment of high FLI. 
It has been reported that exercise training can lead to cardiometabolic benefits, such as reductions in waist circumference, LDL, triglycerides and blood pressure, as well as an increase in HDL $[98,112]$. In the present study, we observed an increment of HDL and glucose. The LDL and triglycerides decreased and blood pressure slightly increased in both groups. The low level of HDL cholesterol is a powerful predictor of the increased cardiovascular risk factor [113]. It must be pointed out that, although the EX and EX + WB-EMS groups experienced reductions in cardiometabolic risk, the improvement observed in the EX + WB-EMS group is clinically greater. According to previous studies, HDL levels are significantly higher in premenopausal and postmenopausal women compared with age-matched men [73]. Therefore, this could be considered as a gender-specific protective factor [114]. Our results show that, while a physical exercise intervention based on the international recommendations (EX group) improved the cardiometabolic score values, EX + WB-EMS improved slightly more, so it can be suggested that the same exercise training with simultaneous WB-EMS could be a suitable complement to enhance those effects. Thus, the present study follows the same line as Schink et al. 2018 [115], who conclude that WB-EMS can be used as a complement to voluntary exercise, for improving fitness and health in healthy subjects and in patients who cannot perform conventional voluntary exercise because of illness.

The glucose levels in this study's baseline would be in concordance with those obtained in previous studies, both in other Spanish regions and other developed countries [116-118]. This pattern is repeated in most European countries, according to studies by the World Health Organization $[119,120]$. The glucose values never exceeded $<100 \mathrm{mg} / \mathrm{dL}$, which is considered to be under healthy parameters [120]. The data indicated that the two groups presented slightly higher levels of glucose than the basal ones, against what could be expected. It could be attributed to a greater hepatic and muscular glycogen capacity of mobilization, which would lead to a better performance in physical activities [118].

Aging, together with usually low estrogen levels, is commonly related to decreases in postmenopausal women's muscle function [121]. This phenomenon is greatly related to impairments in muscle strength [122,123]. The decline in strength in older adults has been linked to impaired ability to perform daily living tasks, including climbing stairs and rising from a chair, together with reduced ability to recover from a trip or a slip [2]. Therefore, strength maintenance is important in fall prevention and independence. Due to this, methodologies that aim the strength enhancement are of extraordinary importance in the postmenopausal woman.

On one hand, throughout the last years, some studies observed successful results in the use of WB-EMS to enhance maximum dynamic and isometric strength in older individuals $[35,39,62,124,125]$. Unfortunately, the corresponding protocols could not guarantee the reliability of the extracted data, showing a high risk of bias [44]. On the hand, Amaro et al. 2019 [45] carried out a 12-week randomized controlled trial with a parallel group, in which one of the groups performed a high-intensity interval training (HIIT group). In the same way as our protocol, their experimental group performed the same training program adding WB-EMS. The authors did not observe significant differences. Taking into account the obvious controversy, as well as the lack of bibliography analyzing the WB-EMS's effects on the strength in the older adults, there is a need for future clinical trials aimed at resolving the problem.

Many of the studies that try to associate physical condition with lifestyle have shown that the level of activity positively influences the strength of the legs, arms and aerobic capacity $[126,127]$. Indeed, strength and aerobic capacity are the two components with a more important influence on people's health and functional independence [112]. There is a quality of evidence related to the implication of strength in the level of autonomy and independence of people $[128,129]$. In detail, lower limb strength has been linked to a better subjective perception of functional status [130]. In the present study, we have found significant improvements in both groups in both leg and arm strength and aerobic resistance. Besides, significant improvements have been observed between groups in 
favor of the EX + WB-EMS group. For this reason, we suggest that a 10-week combined exercise program of strength and resistance with WB-EMS leads to higher improvements than traditional training in postmenopausal women. When women enter the menopause phase, estrogen production decrease and the many beneficial effects of this hormone on cardiovascular health are lost whereby the age-related risk of cardiovascular disease is accelerated [21]. Hormone replacement therapy can, to some extent, counteract the loss of estrogen, but an attractive alternative to hormone therapy is regular physical activity, as it is known that exercise induces many of the same cardiovascular health protective effects as estrogen [21].

The training done in this job seems to be relatively safe. As a result, the prevention of functional deterioration is obtained, which allows a greater capacity to carry out the activities of daily life. It is important to note that no side effects have been reported associated with WB-EMS training. Even though physical performance decreases with aging, the activities carried out in this training program seem to be useful to guarantee higher levels of strength and endurance in postmenopausal women.

The study's findings indicated that there was an association between cardiac risk, FLI, and physical fitness in postmenopausal women, suggesting that physical fitness is associated with better cardiovascular and functional health. However, despite scientific evidence, it should be borne in mind that this training methodology is not accessible to the general population. Knowing the strong association between physical fitness and health parameters, the practice of this type of activity should be promoted by public institutions to specific populations such as postmenopausal women, to improve their health and increase their functional capacity.

The present study has several strengths. Although there is no current established gold standard to determine physical fitness in older adults, the proposed tets have been positioned as one of the most used tools to objectively evaluate functional performance among older adults. Similarly, the fatty liver index and cardiometabolic risk score have been suggested as a more sensitive scale for detecting changes in the individual's biological status than previously validated instruments. As another strength of this study, we should mention that its protocol ensures the assessment of the isolated WB-EMS' effects, since both groups performed the same training program in the intervention.

Our study also has limitations. The nutritional control of the sample was not carried out throughout the treatment, since only instructions regarding keeping on with the usual diet were given to participants. The importance of caloric intake control is necessary for the aiming of body composition and cardiovascular improvements. Finally, in order to estimate the maximum intensity at which the participants could be electrostimulated, a pain threshold test was performed. This could be a parameter that has an excessive subjectivity, so we cannot categorically state that the intensity at which the current was applied was that required to cause adaptations.

\section{Conclusions}

The use of this new training technique has interesting results. The finding of this work suggests that a supervised exercise training intervention (independently of its modality) improves cardiometabolic risk, physical fitness, and fatty liver index in postmenopausal women. Although both groups experienced reductions in cardiometabolic risk, the effect seen in the EX+EMS group could be clinically greater. It should be pointed out that the creatine kinase in the EX + EMS group did not exceed the limits considered normal, indicating the absence of excessive muscle damage. These findings suggest that if the technology is used correctly, the results are positive. Further research is needed to consider the impact of WB-EMS on postmenopausal women.

Author Contributions: Conceptualization, J.R.-M.; methodology, A.P.-R. and J.R.-M.; formal analysis, J.V.B.-G. and V.H.-G.; investigation, V.H.-G.; resources, A.P.-R.; data curation, J.V.B.-G., A.L. and E.S.; writing—original draft preparation, J.R.-M.; writing—review and editing, A.P.-R. and J.V.B.-G.; 
supervision, J.R.-M.; project administration, J.R.-M. All authors have read and agreed to the published version of the manuscript.

Funding: This study was funded by the non-profit organization: Institut de Desenvolupament Social i Territorial. Universitat de Lleida. The name of this project is "Active aging, quality of live and intergenerational relationships". Grant INDEST2016.

Institutional Review Board Statement: The study was conducted according to the guidelines of the Declaration of Helsinki, and approved by the Ethics Committee of Arnau of Vilanova University Hospital, Lléida, (Spain) (CEIC-1701).

Informed Consent Statement: Informed consent was obtained from all subjects involved in the study. Written informed consent has been obtained from the patients to publish this paper.

Data Availability Statement: The spreadsheet is available at https: / / osf.io (Last Updated: 29 June 2020) (Effects of whole-body electromyostimulatin on physical fitness in postmenopausal women: a randomized controlled trial).

Acknowledgments: We thank the Ekke de Lleida gym for the transfer of its material and facilities for the realization of this study and to Wiemspro S.L. for its logistic support.

Conflicts of Interest: The authors declare no conflict of interest.

\section{References}

1. Bullo, V.; Bergamin, M.; Gobbo, S.; Sieverdes, J.C.; Zaccaria, M.; Neunhaeuserer, D.; Ermolao, A. The effects of Pilates exercise training on physical fitness and wellbeing in the elderly: A systematic review for future exercise prescription. Prev. Med. 2015, 75, 1-11. [CrossRef]

2. Fragala, M.S.; Cadore, E.L.; Dorgo, S.; Izquierdo, M.; Kraemer, W.J.; Peterson, M.D.; Ryan, E.D. Resistance Training for Older Adults: Position Statement From the National Strength and Conditioning Association. J. strength Cond. Res. 2019, 33, 2019-2052. [CrossRef]

3. Chughtai, M.; Gwam, C.U.; Mohamed, N.; Khlopas, A.; Sodhi, N.; Sultan, A.A.; Bhave, A.; Mont, M.A. Impact of Physical Activity and Body Mass Index in Cardiovascular and Musculoskeletal Health: A Review. Surg. Technol. Int. 2017, 31, 213-220. [PubMed]

4. Blodgett, J.; Theou, O.; Kirkland, S.; Andreou, P.; Rockwood, K. The association between sedentary behaviour, moderate-vigorous physical activity and frailty in NHANES cohorts. Maturitas 2015, 80, 187-191. [CrossRef] [PubMed]

5. Manas, A.; Del Pozo-Cruz, B.; Guadalupe-Grau, A.; Marin-Puyalto, J.; Alfaro-Acha, A.; Rodriguez-Manas, L.; Garcia-Garcia, F.J.; Ara, I. Reallocating Accelerometer-Assessed Sedentary Time to Light or Moderate- to Vigorous-Intensity Physical Activity Reduces Frailty Levels in Older Adults: An Isotemporal Substitution Approach in the TSHA Study. J. Am. Med. Dir. Assoc. 2018, 19, 185.e1-185.e6. [CrossRef] [PubMed]

6. Reiner, M.; Niermann, C.; Jekauc, D.; Woll, A. Long-term health benefits of physical activity-a systematic review of longitudinal studies. BMC Public Health 2013, 13, 813. [CrossRef] [PubMed]

7. WHO. Global Recommendations on Physical Activity for Health. 2015. Available online: https://www.who.int/ dietphysicalactivity/factsheet_recommendations/en/\#.XpA0CfV9LJE (accessed on 10 April 2020).

8. Sun, F.; Norman, I.J.; While, A.E. Physical activity in older people: A systematic review. BMC Public Health 2013, 13, 449. [CrossRef]

9. Matthews, C.E.; Chen, K.Y.; Freedson, P.S.; Buchowski, M.S.; Beech, B.M.; Pate, R.R.; Troiano, R.P. Amount of time spent in sedentary behaviors in the United States, 2003-2004. Am. J. Epidemiol. 2008, 167, 875-881. [CrossRef]

10. del Pozo-Cruz, B.; Mañas, A.; Martín-García, M.; Marín-Puyalto, J.; García-García, F.J.; Rodriguez-Mañas, L.; Guadalupe-Grau, A.; Ara, I. Frailty is associated with objectively assessed sedentary behaviour patterns in older adults: Evidence from the Toledo Study for Healthy Aging (TSHA). PLoS ONE 2017, 12, e0183911. [CrossRef]

11. Pano-Rodriguez, A.; Beltran-Garrido, J.V.; Hernandez-Gonzalez, V.; Reverter-Masia, J. Effects of Whole-Body Electromyostimulation on Physical Fitness in Postmenopausal Women: A Randomized Controlled Trial. Sensors 2020, 20, 1482. [CrossRef]

12. Fitzpatrick, E.; Dhawan, A. Noninvasive biomarkers in non-alcoholic fatty liver disease: Current status and a glimpse of the future. World J. Gastroenterol. 2014, 20, 10851-10863. [CrossRef]

13. Koot, B.G.P.; van der Baan-Slootweg, O.H.; Bohte, A.E.; Nederveen, A.J.; van Werven, J.R.; Tamminga-Smeulders, C.L.J.; Merkus, M.P.; Schaap, F.G.; Jansen, P.L.M.; Stoker, J.; et al. Accuracy of prediction scores and novel biomarkers for predicting nonalcoholic fatty liver disease in obese children. Obesity 2013, 21, 583-590. [CrossRef] [PubMed]

14. Kahl, S.; Strassburger, K.; Nowotny, B.; Livingstone, R.; Kluppelholz, B.; Kessel, K.; Hwang, J.-H.; Giani, G.; Hoffmann, B.; Pacini, G.; et al. Comparison of liver fat indices for the diagnosis of hepatic steatosis and insulin resistance. PLoS ONE 2014, 9 , e94059. [CrossRef] [PubMed]

15. Lee, J.-H.; Kim, D.; Kim, H.J.; Lee, C.-H.; Yang, J.I.; Kim, W.; Kim, Y.J.; Yoon, J.-H.; Cho, S.-H.; Sung, M.-W.; et al. Hepatic steatosis index: A simple screening tool reflecting nonalcoholic fatty liver disease. Dig. Liver Dis. 2010, 42, 503-508. [CrossRef] 
16. Warburton, D.E.R.; Nicol, C.W.; Bredin, S.S.D. Health benefits of physical activity: The evidence. CMAJ 2006, 174, 801-809. [CrossRef]

17. Zhou, K.; Cen, J. The fatty liver index (FLI) and incident hypertension: A longitudinal study among Chinese population. Lipids Health Dis. 2018, 17, 214. [CrossRef]

18. Balducci, S.; Zanuso, S.; Cardelli, P.; Salvi, L.; Bazuro, A.; Pugliese, L.; Maccora, C.; Iacobini, C.; Conti, F.G.; Nicolucci, A.; et al. Effect of high- versus low-intensity supervised aerobic and resistance training on modifiable cardiovascular risk factors in type 2 diabetes; the Italian Diabetes and Exercise Study (IDES). PLoS ONE 2012, 7, e49297.

19. Paluch, A.E.; Church, T.S.; Blair, S.N. Effect of an Intensive Exercise Intervention Strategy on Modifiable Cardiovascular Risk Factors in Subjects with Type 2 Diabetes Mellitus. Curr. Cardiovasc. Risk Rep. 2011, 5, 481. [CrossRef]

20. Green, D.J.; Hopman, M.T.E.; Padilla, J.; Laughlin, M.H.; Thijssen, D.H.J. Vascular Adaptation to Exercise in Humans: Role of Hemodynamic Stimuli. Physiol. Rev. 2017, 97, 495-528. [CrossRef]

21. Gliemann, L.; Hellsten, Y. The exercise timing hypothesis: Can exercise training compensate for the reduction in blood vessel function after menopause if timed right? J. Physiol. 2019, 597, 4915-4925. [CrossRef] [PubMed]

22. Pierce, G.L.; Donato, A.J.; LaRocca, T.J.; Eskurza, I.; Silver, A.E.; Seals, D.R. Habitually exercising older men do not demonstrate age-associated vascular endothelial oxidative stress. Aging Cell 2011, 10, 1032-1037. [CrossRef]

23. Santos-Parker, J.R.; Strahler, T.R.; Vorwald, V.M.; Pierce, G.L.; Seals, D.R. Habitual aerobic exercise does not protect against microor macrovascular endothelial dysfunction in healthy estrogen-deficient postmenopausal women. J. Appl. Physiol. 2017, 122, 11-19. [CrossRef]

24. McKinney, E.S.; James, S.R.; Murray, S.S.; Nelson, K.A.J. Maternal-Child Nursing; Elsevier: London, UK, $2017 ;$ ISBN 9780323401708.

25. Nyberg, M.; Egelund, J.; Mandrup, C.M.; Nielsen, M.B.; Mogensen, A.S.; Stallknecht, B.; Bangsbo, J.; Hellsten, Y. Early Postmenopausal Phase Is Associated With Reduced Prostacyclin-Induced Vasodilation That Is Reversed by Exercise Training: The Copenhagen Women Study. Hypertension 2016, 68, 1011-1020. [CrossRef]

26. Tunstall-Pedoe, H.; Kuulasmaa, K.; Amouyel, P.; Arveiler, D.; Rajakangas, A.M.; Pajak, A. Myocardial infarction and coronary deaths in the World Health Organization MONICA Project. Registration procedures, event rates, and case-fatality rates in 38 populations from 21 countries in four continents. Circulation 1994, 90, 583-612. [CrossRef]

27. Egelund, J.; Jorgensen, P.G.; Mandrup, C.M.; Fritz-Hansen, T.; Stallknecht, B.; Bangsbo, J.; Nyberg, M.; Hellsten, Y. Cardiac Adaptations to High-Intensity Aerobic Training in Premenopausal and Recent Postmenopausal Women: The Copenhagen Women Study. J. Am. Heart Assoc. 2017, 6, e005469. [CrossRef]

28. Gibala, M.J.; Little, J.P.; Macdonald, M.J.; Hawley, J.A. Physiological adaptations to low-volume, high-intensity interval training in health and disease. J. Physiol. 2012, 590, 1077-1084. [CrossRef]

29. Hwang, C.-L.; Yoo, J.-K.; Kim, H.-K.; Hwang, M.-H.; Handberg, E.M.; Petersen, J.W.; Christou, D.D. Novel all-extremity highintensity interval training improves aerobic fitness, cardiac function and insulin resistance in healthy older adults. Exp. Gerontol. 2016, 82, 112-119. [CrossRef]

30. Osawa, Y.; Azuma, K.; Tabata, S.; Katsukawa, F.; Ishida, H.; Oguma, Y.; Kawai, T.; Itoh, H.; Okuda, S.; Matsumoto, H. Effects of 16-week high-intensity interval training using upper and lower body ergometers on aerobic fitness and morphological changes in healthy men: A preliminary study. Open Access J. Sport. Med. 2014, 5, 257-265. [CrossRef]

31. Sculthorpe, N.F.; Herbert, P.; Grace, F. One session of high-intensity interval training (HIIT) every 5 days, improves muscle power but not static balance in lifelong sedentary ageing men: A randomized controlled trial. Medicine 2017, 96, e6040. [CrossRef]

32. Hurst, C.; Weston, K.L.; Weston, M. The effect of 12 weeks of combined upper- and lower-body high-intensity interval training on muscular and cardiorespiratory fitness in older adults. Aging Clin. Exp. Res. 2019, 31, 661-671. [CrossRef]

33. Dehail, P.; Duclos, C.; Barat, M. Electrical stimulation and muscle strengthening. Ann. Readapt. Med. Phys. 2008, 51, 441-451. [CrossRef]

34. Wiemspro-Electrostimulation for Fitness. Available online: https: / / wiemspro.com (accessed on 22 July 2019).

35. Kemmler, W.; Bebenek, M.; Engelke, K.; von Stengel, S. Impact of whole-body electromyostimulation on body composition in elderly women at risk for sarcopenia: The Training and ElectroStimulation Trial (TEST-III). Age 2014, 36, 395-406. [CrossRef]

36. Filipovic, A.; Kleinoder, H.; Pluck, D.; Hollmann, W.; Bloch, W.; Grau, M.; Kleinöder, H.; Plück, D.; Hollmann, W.; Bloch, W.; et al. Influence of Whole-Body Electrostimulation on Human Red Blood Cell Deformability. J. Strength Cond. Res. 2015, 29, $2570-2578$. [CrossRef]

37. Dörmann, U.; Wirtz, N.; Micke, F.; Morat, M.; Kleinöder, H.; Donath, L. The Effects of Superimposed Whole-Body Electromyostimulation During Short-Term Strength Training on Physical Fitness in Physically Active Females: A Randomized Controlled Trial. Front. Physiol. 2019, 10, 728. [CrossRef] [PubMed]

38. Kemmler, W.; Grimm, A.; Bebenek, M.; Kohl, M.; von Stengel, S.; Weissenfels, A.; Teschler, M.; Willert, S.; Bebenek, M.; Shojaa, M.; et al. Effects of Combined Whole-Body Electromyostimulation and Protein Supplementation on Local and Overall Muscle/Fat Distribution in Older Men with Sarcopenic Obesity: The Randomized Controlled Franconia Sarcopenic Obesity (FranSO) Study. Calcif. Tissue Int. 2018, 103, 266-277. [CrossRef] [PubMed]

39. Kemmler, W.; Weissenfels, A.; Teschler, M.; Willert, S.; Bebenek, M.; Shojaa, M.; Kohl, M.; Freiberger, E.; Sieber, C.; von Stengel, S. Whole-body electromyostimulation and protein supplementation favorably affect sarcopenic obesity in community-dwelling older men at risk: The randomized controlled FranSO study. Clin. Interv. Aging 2017, 12, 1503-1513. [CrossRef] [PubMed] 
40. Micke, F.; Kleinöder, H.; Dörmann, U.; Wirtz, N.; Donath, L. Effects of an Eight-Week Superimposed Submaximal Dynamic Whole-Body Electromyostimulation Training on Strength and Power Parameters of the Leg Muscles: A Randomized Controlled Intervention Study. Front. Physiol. 2018, 9, 1719. [CrossRef] [PubMed]

41. Filipovic, A.; DeMarees, M.; Grau, M.; Hollinger, A.; Seeger, B.; Schiffer, T.; Bloch, W.; Gehlert, S. Superimposed Whole-Body Electrostimulation Augments Strength Adaptations and Type II Myofiber Growth in Soccer Players During a Competitive Season. Front. Physiol. 2019, 10, 1187. [CrossRef] [PubMed]

42. Wittmann, K.; Sieber, C.; Von Stengel, S.; Kohl, M.; Freiberger, E.; Jakob, F.; Lell, M.; Engelke, K.; Kemmler, W. Impact of whole body electromyostimulation on cardiometabolic risk factors in older women with sarcopenic obesity: The randomized controlled FORMOsA-sarcopenic obesity study. Clin. Interv. Aging 2016, 11, 1697-1706. [CrossRef]

43. Kemmler, W.; Kohl, M.; Freiberger, E.; Sieber, C.; von Stengel, S. Effect of whole-body electromyostimulation and/or protein supplementation on obesity and cardiometabolic risk in older men with sarcopenic obesity: The randomized controlled FranSO trial. BMC Geriatr. 2018, 18, 70. [CrossRef] [PubMed]

44. Pano-Rodriguez, A.; Beltran-Garrido, J.V.; Hernández-González, V.; Reverter-Masia, J. Effects of whole-body ELECTROMYOSTIMULATION on health and performance: A systematic review. BMC Complement. Altern. Med. 2019, 19, 87. [CrossRef] [PubMed]

45. Amaro-gahete, F.J.; Delao, A.; Jurado-fasoli, L.; Dote-montero, M.; Gutiérrez, Á.; Ruiz, J.R.; Castillo, M.J. Changes in Physical Fitness After 12 Weeks of Structured Concurrent Exercise Training, High Intensity Interval Training, or Whole-Body Electromyostimulation Training in Sedentary Middle-Aged Adults: A Randomized Controlled Trial. Front. Physiol. 2019, 10, 1-15. [CrossRef] [PubMed]

46. Pano-Rodriguez, A.; Beltran-Garrido, J.V.; Hernandez-Gonzalez, V.; Reverter-Masia, J. Effects of Whole Body Electromyostimulation on Physical Fitness and Health in Postmenopausal Women: A Study Protocol for a Randomized Controlled Trial. Front. Public Health 2020, 8, 313. [CrossRef]

47. Pano-Rodriguez, A.; Beltran-Garrido, J.V.; Hernandez-Gonzalez, V.; Nasarre-Nacenta, N.; Reverter-Masia, J. Impact of Whole Body Electromyostimulation on Velocity, Power and Body Composition in Postmenopausal Women: A Randomized Controlled Trial. Int. J. Environ. Res. Public Health 2020, 17, 4982. [CrossRef]

48. Schulz, K.F.; Altman, D.G.; Moher, D. CONSORT 2010 Statement: Updated guidelines for reporting parallel group randomised trials. BMJ 2010, 340, 18-27. [CrossRef]

49. Moniker Privacy Services Random Team Generator-Split a List into Random Groups. Available online: https://www. randomlists.com/team-generator (accessed on 26 November 2019).

50. Sjöström, M.; Oja, P.; Hagströmer, M.; Smith, B.J.; Bauman, A. Health-enhancing physical activity across European Union countries: The Eurobarometer study. J. Public Health 2006, 14, 291-300. [CrossRef]

51. Kovanen, V.; Aukee, P.; Kokko, K.; Finni, T.; Tarkka, I.M.; Tammelin, T.; Kujala, U.M.; Sipilä, S.; Laakkonen, E.K. Design and protocol of Estrogenic Regulation of Muscle Apoptosis (ERMA) study with 47 to 55-year-old women's cohort: Novel results show menopause-related differences in blood count. Menopause 2018, 25, 1020-1032. [CrossRef]

52. Aragão-Santos, J.C.; de Resende-Neto, A.G.; Costa Nogueira, A.; Feitosa-Neta, M.L.; Albuquerque Brandão, L.H.; da Silva Chaves, L.M.; da Silva-Grigoletto, M.E. The effects of functional and traditional strength training on different parameters of strength elderly women: A trial randomized and controlled. J. Sport. Med. Phys. Fit. 2018, 59, 380-386. [CrossRef]

53. Gonzalez-Badillo, J.J.; Sanchez-Medina, L. Movement velocity as a measure of loading intensity in resistance training. Int. J. Sports Med. 2010, 31, 347-352. [CrossRef]

54. Wirtz, N.; Wahl, P.; Kleinöder, H.; Wechsler, K.; Achtzehn, S.; Mester, J.; Kleinder, H.; Wechsler, K.; Achtzehn, S.; Mester, J. Acute metabolic, hormonal, and psychological responses to strength training with superimposed EMS at the beginning and the end of a 6 week training period. J. Musculoskelet. Neuronal Interact. 2015, 15, 325-332.

55. Reed, J.L.; Pipe, A.L. The talk test: A useful tool for prescribing and monitoring exercise intensity. Curr. Opin. Cardiol. 2014, 29, 475-480. [CrossRef]

56. Borg, G. Perceived exertion as an indicator of somatic stress. Scand. J. Rehabil. Med. 1970, 2, 92-98.

57. Filipovic, A.; Klein Der, H.; Rmann, U.D.; Mester, J. Electromyostimulation-a Systematic Review of the Influence of Training Regimens and Stimulation Parameters on Effectiveness in Electromyostimulation Training of Selected Strength Parameters. J. strength Cond. Res. 2011, 11, 3218-3238. [CrossRef] [PubMed]

58. Doucet, B.M.; Lam, A.; Griffin, L. Neuromuscular electrical stimulation for skeletal muscle function. Yale J. Biol. Med. 2012, 85, 201-215. Available online: http:/ / www.pubmedcentral.nih.gov/articlerender.fcgi?artid=3375668\&tool=pmcentrez\&rendertype= abstract (accessed on 3 June 2021). [PubMed]

59. A Guide to the Use of Electrical Stimulation in Pediatric Nurodisbility/Association of Paediatric Chartered Physiotherapists. Available online: https:/ / apcp.csp.org.uk/content/guide-use-electrical-stimulation-paedaitric-neurodisability (accessed on 19 December 2019).

60. Deley, G.; Babault, N. Could low-frequency electromyostimulation training be an effective alternative to endurance training? An overview in one adult. J. Sport. Sci. Med. 2014, 13, 444-450.

61. Petrofsky, J. The effect of the subcutaneous fat on the transfer of current through skin and into muscle. Med. Eng. Phys. 2008, 30, 1168-1176. [CrossRef] 
62. Kemmler, W.; Schliffka, R.; Mayhew, J.L.; von Stengel, S. Effects of whole-body electromyostimulation on resting metabolic rate, body composition, and maximum strength in postmenopausal women: The Training and ElectroStimulation Trial. J. strength Cond. Res. 2010, 24, 1880-1887. [CrossRef]

63. Alvero Cruz, J.E. Al Protocolo de valoración de la composición corporal para el reconocimiento médico-deportivo. Documento de Consenso del Grupo Español de Cineantropometría de la Federacion Española de Medicina del Deporte. Arch. Med. Deport. 2009, 26, 166-179.

64. International Society for Advancement of Kinanthropometry. International Strandars for Anthropometric Assessment: ISAK; International Society for Advancement of Kinanthropometry: Potchefstroom, South Africa, 2001.

65. Whelton, P.K.; Williams, B. The 2018 European Society of Cardiology/European Society of Hypertension and 2017 American College of Cardiology/American Heart Association Blood Pressure Guidelines: More Similar Than Different. JAMA 2018, 320, 1749-1750. [CrossRef] [PubMed]

66. Stefanska, A.; Bergmann, K.; Sypniewska, G. Metabolic Syndrome and Menopause: Pathophysiology, Clinical and Diagnostic Significance. Adv. Clin. Chem. 2015, 72, 1-75.

67. Onat, A.; Karadeniz, Y.; Tusun, E.; Yuksel, H.; Kaya, A. Advances in understanding gender difference in cardiometabolic disease risk. Expert Rev. Cardiovasc. Ther. 2016, 14, 513-523. [CrossRef]

68. Bedogni, G.; Bellentani, S.; Miglioli, L.; Masutti, F.; Passalacqua, M.; Castiglione, A.; Tiribelli, C. The Fatty Liver Index: A simple and accurate predictor of hepatic steatosis in the general population. BMC Gastroenterol. 2006, 6, 33. [CrossRef]

69. Huang, X.; Xu, M.; Chen, Y.; Peng, K.; Huang, Y.; Wang, P.; Ding, L.; Lin, L.; Xu, Y.; Chen, Y.; et al. Validation of the Fatty Liver Index for Nonalcoholic Fatty Liver Disease in Middle-Aged and Elderly Chinese. Medicine 2015, 94, e1682. [CrossRef] [PubMed]

70. Sagarra-Romero, L.; Vicente-Rodriguez, G.; Pedrero-Chamizo, R.; Vila-Maldonado, S.; Gusi, N.; Villa-Vicente, J.G.; Espino, L.; Gonzalez-Gross, M.; Casajus, J.A.; Ara, I.; et al. Is Sitting Time Related with Physical Fitness in Spanishelderly Population? The EXERNET Multicenter Study. J. Nutr. Health Aging 2019, 23, 401-407. [CrossRef] [PubMed]

71. Colado, J.C.; Triplett, N.T. Effects of a short-term resistance program using elastic bands versus weight machines for sedentary middle-aged women. J. Strength Cond. Res. 2008, 22, 1441-1448. [CrossRef]

72. Bajerska, J.; Chmurzynska, A.; Muzsik, A.; Krzyzanowska, P.; Madry, E.; Malinowska, A.M.; Walkowiak, J. Weight loss and metabolic health effects from energy-restricted Mediterranean and Central-European diets in postmenopausal women: A randomized controlled trial. Sci. Rep. 2018, 8, 11170. [CrossRef]

73. Aranda-Ramírez, P.; López-Jurado, M.; Llopis-González, J.; Ruiz-Cabello-Turmo, P.; del Mar Fernández-Martínez, M. The effectiveness of an education program and nutritional intervention, integrated health and physical activity in postmenopausal women. Nutr. Hosp. 2016, 33, 359-367.

74. Mataix, J.; Lopez-Frias, M.; Martinez-de-Victoria, E.; Lopez-Jurado, M.; Aranda, P.; Llopis, J. Factors associated with obesity in an adult Mediterranean population: Influence on plasma lipid profile. J. Am. Coll. Nutr. 2005, 24, 456-465. [CrossRef]

75. Cornellana, M.J.; Harvey, X.; Carballo, A.; Khartchenko, E.; Llaneza, P.; Palacios, S.; Mendoza, N. Sexual health in Spanish postmenopausal women presenting at outpatient clinics. Climacteric 2017, 20, 164-170. [CrossRef]

76. Hita-Contreras, F.; Zagalaz-Anula, N.; Martinez-Amat, A.; Cruz-Diaz, D.; Sanchez-Montesinos, I.; Aibar-Almazan, A.; LomasVega, R. Sleep quality and its association with postural stability and fear of falling among Spanish postmenopausal women. Menopause 2018, 25, 62-69. [CrossRef]

77. Keating, S.E.; Machan, E.A.; O'Connor, H.T.; Gerofi, J.A.; Sainsbury, A.; Caterson, I.D.; Johnson, N.A. Continuous exercise but not high intensity interval training improves fat distribution in overweight adults. J. Obes. 2014, 2014, 834865. [CrossRef]

78. Olinto, M.T.A.; Nacul, L.C.; Gigante, D.P.; Costa, J.S.D.; Menezes, A.M.B.; Macedo, S. Waist circumference as a determinant of hypertension and diabetes in Brazilian women: A population-based study. Public Health Nutr. 2004, 7, 629-635. [CrossRef]

79. Fox, C.S.; Massaro, J.M.; Hoffmann, U.; Pou, K.M.; Maurovich-Horvat, P.; Liu, C.-Y.; Vasan, R.S.; Murabito, J.M.; Meigs, J.B.; Cupples, L.A.; et al. Abdominal visceral and subcutaneous adipose tissue compartments: Association with metabolic risk factors in the Framingham Heart Study. Circulation 2007, 116, 39-48. [CrossRef] [PubMed]

80. Kemmler, W.; Birlauf, A. Einfluss eines Elektromyostimulations-trainings auf die körperzusammensetzung bei älteren männern mit metabolischem syndrom. Dtsch. Z. Sportmed. 2010, 61, 117-123.

81. Kemmler, W.; Birlauf, A.; von Stengel, S. Effects of Whole-Body-Electromyostimulation on Body Composition and Cardiac Risk Factors in Elderly Men with the Metabolic Syndrome. The TEST-II Study. Dtsch. Z. Sportmed. 2010, 61, 117-123.

82. Kemmler, W.; von Stengel, S.; Engelke, K.; Häberle, L.; Mayhew, J.L.; Kalender, W.A. Exercise, Body Composition, and Functional Ability. A Randomized Controlled Trial. Am. J. Prev. Med. 2010, 38, 279-287. [CrossRef]

83. Kemmler, W.; Schliffka, R.; Mayhew, J.L.; von Stengel, S. Effects of whole body electromyostimulation on resting metabolic rate, body composition in postmenopausal women. J. Strength Cond. Res. 2013, 7, 1880-1887.

84. Kemmler, W.; Teschler, M.; Weißenfels, A.; Bebenek, M.; Fröhlich, M.; Kohl, M.; von Stengel, S.; Weissenfels, A.; Bebenek, M.; Frohlich, M.; et al. Effects of whole-body electromyostimulation versus high-intensity resistance exercise on body composition and strength: A randomized controlled study. Evid. Based Complement. Altern. Med. 2016, 2016, 9236809. [CrossRef]

85. Filipovic, A.; Grau, M.; Kleinoeder, H.; Zimmer, P.; Hollmann, W.; Bloch, W. Effects of a Whole-Body Electrostimulation Program on Strength, Sprinting, Jumping, and Kicking Capacity in Elite Soccer Players. J. Sport. Sci. Med. 2016, 15, 639-648.

86. Wirtz, N.; Zinner, C.; Doermann, U.; Kleinoeder, H.; Mester, J. Effects of Loaded Squat Exercise with and without Application of Superimposed EMS on Physical Performance. J. Sport. Sci. Med. 2016, 15, $26-33$. 
87. Pescatello, L.S.; MacDonald, H.V.; Lamberti, L.; Johnson, B.T. Exercise for Hypertension: A Prescription Update Integrating Existing Recommendations with Emerging Research. Curr. Hypertens. Rep. 2015, 17, 87. [CrossRef]

88. Whelton, S.P.; Chin, A.; Xin, X.; He, J. Effect of aerobic exercise on blood pressure: A meta-analysis of randomized, controlled trials. Ann. Intern. Med. 2002, 136, 493-503. [CrossRef]

89. Dimeo, F.; Pagonas, N.; Seibert, F.; Arndt, R.; Zidek, W.; Westhoff, T.H. Aerobic exercise reduces blood pressure in resistant hypertension. Hypertens 2012, 60, 653-658. [CrossRef] [PubMed]

90. Kastner, A.; Braun, M.; Meyer, T. Two Cases of Rhabdomyolysis After Training With Electromyostimulation by 2 Young Male Professional Soccer Players. Clin. J. Sport Med. Off. J. Can. Acad. Sport Med. 2015, 25, e71-e73. [CrossRef] [PubMed]

91. Stollberger, C.; Finsterer, J. Side effects of and contraindications for whole-body electro-myo-stimulation: A viewpoint. BMJ Open Sport Exerc. Med. 2019, 5, e000619. [CrossRef]

92. Hong, J.Y.; Hyeok Oh, J.; Shin, J.-H. Rhabdomyolysis caused by knee push-ups with whole body electromyostimulation. Br. J. Hosp. Med. 2016, 77, 542-543. [CrossRef] [PubMed]

93. Teschler, M.; Mooren, F.C. (Whole-Body) Electromyostimulation, Muscle Damage, and Immune System: A Mini Review. Front. Physiol. 2019, 10, 1461. [CrossRef] [PubMed]

94. Teschler, M.; Weissenfels, A.; Froehlich, M.; Kohl, M.; Bebenek, M.; von Stengel, S.; Kemmler, W. (Very) high creatine kinase (CK) levels after Whole-Body Electromyostimulation. Are there implications for health? Int. J. Clin. Exp. Med. 2016, 9, 22841-22850.

95. Castagno, E.; Lupica, M.; Viola, S.; Savino, F.; Miniero, R. Creatin-kinase elevation after accidental ingestion of almotriptan in an 18-month-old girl. Minerva Pediatr. 2014, 66, 95-97.

96. Khan, F.Y. Rhabdomyolysis: A review of the literature. Neth. J. Med. 2009, 67, 272-283.

97. Quiroga, E.V.; Arze, S.A. Rabdomiolisis, mioglobinuria e injuria renal aguda inducida por el ejercicio: Reporte de un caso en el Centro Médico Boliviano Belga. Gac. Médica Boliv. 2014, 37, 27-30.

98. Arslan, E.; Can, S.; Demirkan, E. Effect of short-term aerobic and combined training program on body composition, lipids profile and psychological health in premenopausal women. Sci. Sports 2017, 32, 106-113. [CrossRef]

99. Del Pozo-Cruz, J.; Magaña, M.; Ballesteros, M.; Porras, M.; Bíes, E.R.; Navas, P.; López-Lluch, G. Influencia de la capacidad funcional sobre el perfil lipídico, daño muscular y perfil bioquímico en personas mayores no institucionalizadas. Rev. Andaluza Med. Deport. 2013, 6, 57-65. [CrossRef]

100. Wang, Y.; Xu, D. Effects of aerobic exercise on lipids and lipoproteins. Lipids Health Dis. 2017, 16, 132. [CrossRef]

101. Zhang, Y.; Lu, D.; Wang, R.; Fu, W.; Zhang, S. Relationship between Muscle Mass/Strength and Hepatic Fat Content in Post-Menopausal Women. Medicina 2019, 55, 629. [CrossRef]

102. Wang, C.; Bai, L. Sarcopenia in the elderly: Basic and clinical issues. Geriatr. Gerontol. Int. 2012, 12, 388-396. [CrossRef]

103. Wong, V.W.-S.; Wong, G.L.-H.; Yip, G.W.-K.; Lo, A.O.-S.; Limquiaco, J.; Chu, W.C.-W.; Chim, A.M.-L.; Yu, C.-M.; Yu, J.; Chan, F.K.-L.; et al. Coronary artery disease and cardiovascular outcomes in patients with non-alcoholic fatty liver disease. Gut 2011, 60, 1721-1727. [CrossRef]

104. Musso, G.; Gambino, R.; Cassader, M.; Pagano, G. Meta-analysis: Natural history of non-alcoholic fatty liver disease (NAFLD) and diagnostic accuracy of non-invasive tests for liver disease severity. Ann. Med. 2011, 43, 617-649. [CrossRef]

105. Sung, K.-C.; Kim, S.H. Interrelationship between fatty liver and insulin resistance in the development of type 2 diabetes. J. Clin. Endocrinol. Metab. 2011, 96, 1093-1097. [CrossRef]

106. Banitalebi, E.; Faramarzi, M.; Nasiri, S.; Mardaniyan, M.; Rabiee, V. Effects of different exercise modalities on novel hepatic steatosis indices in overweight women with type 2 diabetes. Clin. Mol. Hepatol. 2019, 25, 294-304. [CrossRef]

107. Gastaldelli, A.; Kozakova, M.; Hojlund, K.; Flyvbjerg, A.; Favuzzi, A.; Mitrakou, A.; Balkau, B. Fatty liver is associated with insulin resistance, risk of coronary heart disease, and early atherosclerosis in a large European population. Hepatology 2009, 49, 1537-1544. [CrossRef] [PubMed]

108. Arteaga, I.; Buezo, I.; Exposito, C.; Pera, G.; Rodriguez, L.; Aluma, A.; Auladell, M.A.; Toran, P.; Caballeria, L. Non-invasive markers of fibrosis in the diagnosis of non-alcoholic fatty liver disease. Gastroenterol. Hepatol. 2014, 37, 503-510. [CrossRef] [PubMed]

109. Balducci, S.; Cardelli, P.; Pugliese, L.; D’Errico, V.; Haxhi, J.; Alessi, E.; Iacobini, C.; Menini, S.; Bollanti, L.; Conti, F.G.; et al. Volume-dependent effect of supervised exercise training on fatty liver and visceral adiposity index in subjects with type 2 diabetes The Italian Diabetes Exercise Study (IDES). Diabetes Res. Clin. Pract. 2015, 109, 355-363. [CrossRef]

110. Barsalani, R.; Riesco, E.; Lavoie, J.-M.; Dionne, I.J. Effect of exercise training and isoflavones on hepatic steatosis in overweight postmenopausal women. Climacteric 2013, 16, 88-95. [CrossRef]

111. Byambasukh, O.; Zelle, D.; Corpeleijn, E. Physical Activity, Fatty Liver, and Glucose Metabolism Over the Life Course: The Lifelines Cohort. Am. J. Gastroenterol. 2019, 114, 907-915. [CrossRef]

112. Rossi, F.E.; Fortaleza, A.C.S.; Neves, L.M.; Buonani, C.; Picolo, M.R.; Diniz, T.A.; Kalva-Filho, C.A.; Papoti, M.; Lira, F.S.; Freitas Junior, I.F. Combined Training (Aerobic Plus Strength) Potentiates a Reduction in Body Fat but Demonstrates No Difference on the Lipid Profile in Postmenopausal Women When Compared With Aerobic Training With a Similar Training Load. J. Strength Cond. Res. 2016, 30, 226-234. [CrossRef]

113. Amaro-Gahete, F.J.; De-la-O, A.; Jurado-Fasoli, L.; Martinez-Tellez, B.; Ruiz, J.R.; Castillo, M.J. Exercise Training as a Treatment for Cardiometabolic Risk in Sedentary Adults: Are Physical Activity Guidelines the Best Way to Improve Cardiometabolic Health? The FIT-AGEING Randomized Controlled Trial. J. Clin. Med. 2019, 8, 2097. [CrossRef] 
114. Bondarev, D.; Laakkonen, E.K.; Finni, T.; Kokko, K.; Kujala, U.M.; Aukee, P.; Kovanen, V.; Sipila, S. Physical performance in relation to menopause status and physical activity. Menopause 2018, 25, 1432-1441. [CrossRef]

115. Schink, K.; Herrmann, H.J.; Schwappacher, R.; Meyer, J.; Orlemann, T.; Waldmann, E.; Wullich, B.; Kahlmeyer, A.; Fietkau, R.; Lubgan, D.; et al. Effects of whole-body electromyostimulation combined with individualized nutritional support on body composition in patients with advanced cancer: A controlled pilot trial. BMC Cancer 2018, 18, 1-17. [CrossRef]

116. Orgaz Gallego, M.P.; Bermejo Lopez, P.; Tricio Armero, M.A.; Abellan Aleman, J.; Solera Albero, J.; Tarraga Lopez, P.J. Metabolic Syndrome and its Components in Spanish Postmenopausal Women. Nutr. Hosp. 2015, 32, 656-666.

117. Cuadros, J.L.; Fernandez-Alonso, A.M.; Cuadros, A.M.; Chedraui, P.; Perez-Lopez, F.R. Body mass index and its correlation to metabolic and hormone parameters in postmenopausal Spanish women. Gynecol. Endocrinol. 2011, 27, 678-684. [CrossRef]

118. Hallajzadeh, J.; Khoramdad, M.; Izadi, N.; Karamzad, N.; Almasi-Hashiani, A.; Ayubi, E.; Qorbani, M.; Pakzad, R.; Hasanzadeh, A.; Sullman, M.J.M.; et al. Metabolic syndrome and its components in premenopausal and postmenopausal women: A comprehensive systematic review and meta-analysis on observational studies. Menopause 2018, 25, 1155-1164. [CrossRef]

119. WHO. Diet, Nutrition and the Prevention of Chronic Diseases Report of the Joint WHO/FAO Expert Consultation. 2014. Available online: https:/ / www.who.int/dietphysicalactivity/publications/trs916/intro/en/\#.XpG6E15gunU (accessed on 11 April 2020).

120. WHO/Europe. Data and Evidence-European Health for All Family of Databases (HFA-DB). Available online: http://www.euro. who.int/en/data-and-evidence/databases/european-health-for-all-family-of-databases-hfa-db (accessed on 11 April 2020).

121. Messier, V.; Rabasa-Lhoret, R.; Barbat-Artigas, S.; Elisha, B.; Karelis, A.D.; Aubertin-Leheudre, M. Menopause and sarcopenia: A potential role for sex hormones. Maturitas 2011, 68, 331-336. [CrossRef] [PubMed]

122. Trombetti, A.; Reid, K.F.; Hars, M.; Herrmann, F.R.; Pasha, E.; Phillips, E.M.; Physiology, E.; Human, J.M. Age-associated declines in muscle mass, strength, power, and physical performance: Impact on fear of falling and quality of life. Osteoporos. Int. 2016, 27, 463-471. [CrossRef]

123. McKinnon, N.B.; Connelly, D.M.; Rice, C.L.; Hunter, S.W.; Doherty, T.J. Neuromuscular contributions to the age-related reduction in muscle power: Mechanisms and potential role of high velocity power training. Ageing Res. Rev. 2017, 35, 147-154. [CrossRef]

124. Kemmler, W.; von Stengel, S. Whole-body electromyostimulation as a means to impact muscle mass and abdominal body fat in lean, sedentary, older female adults: Subanalysis of the TEST-III trial. Clin. Interv. Aging 2013, 8, 1353-1364. [CrossRef]

125. Von Stengel, S.; Bebenek, M.; Engelke, K.; Kemmler, W. Whole-body electromyostimulation to fight osteopenia in elderly females: The randomized controlled training and electrostimulation trial (TEST-III). J. Osteoporos. 2015, 2015, 643520. [CrossRef]

126. Eskurza, I.; Donato, A.J.; Moreau, K.L.; Seals, D.R.; Tanaka, H. Changes in maximal aerobic capacity with age in endurance-trained women: 7-yr follow-up. J. Appl. Physiol. 2002, 92, 2303-2308. [CrossRef]

127. Bouchard, D.R.; Heroux, M.; Janssen, I. Association between muscle mass, leg strength, and fat mass with physical function in older adults: Influence of age and sex. J. Aging Health 2011, 23, 313-328. [CrossRef]

128. Ruiz, J.R.; Segura-Jimenez, V.; Ortega, F.B.; Alvarez-Gallardo, I.C.; Camiletti-Moiron, D.; Aparicio, V.A.; Carbonell-Baeza, A.; Femia, P.; Munguia-Izquierdo, D.; Delgado-Fernandez, M. Objectively measured sedentary time and physical activity in women with fibromyalgia: A cross-sectional study. BMJ Open 2013, 3, e002722. [CrossRef] [PubMed]

129. Alvarez-Gallardo, I.C.; Carbonell-Baeza, A.; Segura-Jimenez, V.; Soriano-Maldonado, A.; Intemann, T.; Aparicio, V.A.; Estevez-Lopez, F.; Camiletti-Moiron, D.; Herrador-Colmenero, M.; Ruiz, J.R.; et al. Physical fitness reference standards in fibromyalgia: The al-Andalus project. Scand. J. Med. Sci. Sports 2017, 27, 1477-1488. [CrossRef] [PubMed]

130. Gowans, S.E.; deHueck, A. Pool exercise for individuals with fibromyalgia. Curr. Opin. Rheumatol. 2007, 19, 168-173. [CrossRef] [PubMed] 